\title{
THE EFFECT OF COUNT, TWIST, OPENING ROLLER SPEED AND ROTOR SPEED ON YARN TENSILE PROPERTIES OF ROTOR SPUN YARNS ${ }^{1}$
}

\author{
Jacquirine Mirembe, Josphat Igadwa Mwasiagi* \\ Department of Manufacturing, Industrial and Textile Engineering, School of Engineering, Moi \\ University, Eldoret, Kenya. \\ igadwa@gmail.com* (corresponding author)
}

\begin{abstract}
Tensile properties are some of the important parameters when considering the quality of rotor spun yarns. This research work set out to investigating the effect of four factors namely count, twist, opening roller speed and rotor speed on the tensile properties of rotor spun yarn. Taguchi Experimental Design was used to study the influence of the four factors on yarn tensile (strength and elongation) properties. The experiments were carried out in a textile factory in Uganda. The results obtained in this research work indicated that both single and combined effects of the selected factors significantly affected yarn strength and elongation. For the given cotton lint, optimum tensile properties could be obtained at $15 \mathrm{Ne}$, 5TM, $8000 \mathrm{rpm}$ and 90,0000 rpm for count, twist, opening roller speed and rotor speed respectively.
\end{abstract}

Keywords: Cotton fiber, yarn, tensile, count, twist, opening roller speed, rotor speed.

\section{Introduction}

The rotor spinning process involves opening and cleaning of cotton lint in blowroom and carding. Further lint processing, includes more opening, cleaning and drafting using drawframes and rotor spinning machine (see Figure 1) is done to get the final yarn product. At the rotor spinning machine, the input material is the drawframe sliver, which is guided by the feed roller and opened up by the opening roller into individual fibers. The separated fibers are removed from the opening roller by air suction through the fiber transport channel. For satisfactory yarn properties, the feed sliver should be free from impurities and hooks so as to avoid clogging the rotor machine [1-2]. After fiber opening the fiber needs to be twisted. The rotor generates the twist as the yarn is carried through each revolution. Lord [2] reported that to produce an open-end yarn, it is necessary to use a very high draft so that the fiber flow is

\footnotetext{
${ }^{1}$ This research was suppoted by METEGA project (www.metega.com)
} 
reduced to just a few fibers in the cross-section. This prevents twist from running back into the fiber supply thereby producing false twist, which would otherwise distort the spinning process.

Rotor spun yarn can be characterized by measuring yarn tensile properties (strength and elongation), yarn evenness and imperfection (neps, thick and thin places). The factors which affect the tensile properties of cotton rotor spun yarns include, cotton fiber properties, yarn properties and machine parameters. A study by Arain et al [3] reported that yarn strength increased with increase of rotor speed, yarn twist and linear density. This could be due to the formation of more wrapping fibers, increased centrifugal force in the spinning region, increased fiber in the yarn cross section and increased fiber interaction [3-5]. Yarn elongation however decreased with increase in rotor speed, but increased with an increase of yarn twist and linear density. Furter [6] stated that yarn elongation exhibited an inverse relationship with linear density. Considering rotor speed, higher speeds creates a strain in the yarn which in return reduces elongation. Other factors which affect yarn tensile properties of rotor spun yarn include preparation of the feed material and opening roller speed [7]. Therefore for a given cotton lint, the spinner needs to consider a wide variety of cotton properties, yarn and machine parameters. The general approach is to consider a few selected factors and then keep the other factors constant as an investigation is carried out on the quality parameters of rotor spun yarns. Ahmed et al [8] considered the effect of rotor speed and count on the properties of rotor spun yarns, while rotor speed, twist and count were considered by Arain et al [3]. Most researchers have considered rotor speed, opening roller speed, rotor diameter and carding conditions, but all considered at least one value at a time or at most three factors [5-6, 9]. While previous works have given an insight on the influence of rotor spinning parameters on rotor spun yarns more investigations needs to be done so as to further improve the quality properties of rotor spun yarns. This study therefore aimed at investigating the effect of opening roller speed, rotor speed, count and twist on yarn tensile properties for cotton yarn. The experiments were carried out in a spinning department of a textile factory in Uganda.

\section{Materials and methods}

Cotton lint and rotor spun yarns were collected from a textile factory in Uganda. The lint samples, which had been procured from Kitgum District of Uganda were characterized under standard conditions using an HVI instrument as per ASTM D5867-2005 standard and the results are given in Table 1. The processing of the cotton yarn included blow room, carding, breaker drawframe and finisher drawframe so as to produce a sliver to be fed to rotor spinning machine for yarn production. The machines used during the manufacture of the yarn are shown in figure 1.

Table 1. Cotton fiber properties

\begin{tabular}{|c|c|}
\hline Fiber Property & Mean \\
\hline Micronaire (micro gram/inch) & 4.3 \\
\hline Maturity & 0.86 \\
\hline Length (mm) & 28.54 \\
\hline Uniformity Index (\%) & 83 \\
\hline Short Fiber Content (\%) & 6.7 \\
\hline Strength (cN/tex) & 28.6 \\
\hline Elongation (\%) & 7 \\
\hline Reflectance (\%) & 74.6 \\
\hline Yellowness (degree) & 10.91 \\
\hline
\end{tabular}




\begin{tabular}{|c|c|}
\hline Trash Content & 26 \\
\hline Trash Area (\%) & 0.45 \\
\hline
\end{tabular}

The rotors used were hybrid G.333/T.333 with a diameter of $34.5 \mathrm{~mm}$, Taguchi experimental design was used to design 18 experiments as shown in Table 2. The count range was selected based on the factory requirements of $\mathrm{Ne} 15$ and 24 for its weaving needs. All other factors were selected based on previous factory values, with allowances for high and low values so as to enable a study of the effect of these factors on tensile properties. Ten yarn packages per each experimental design were spun and tested as per ASTM D2256-2002 standard.

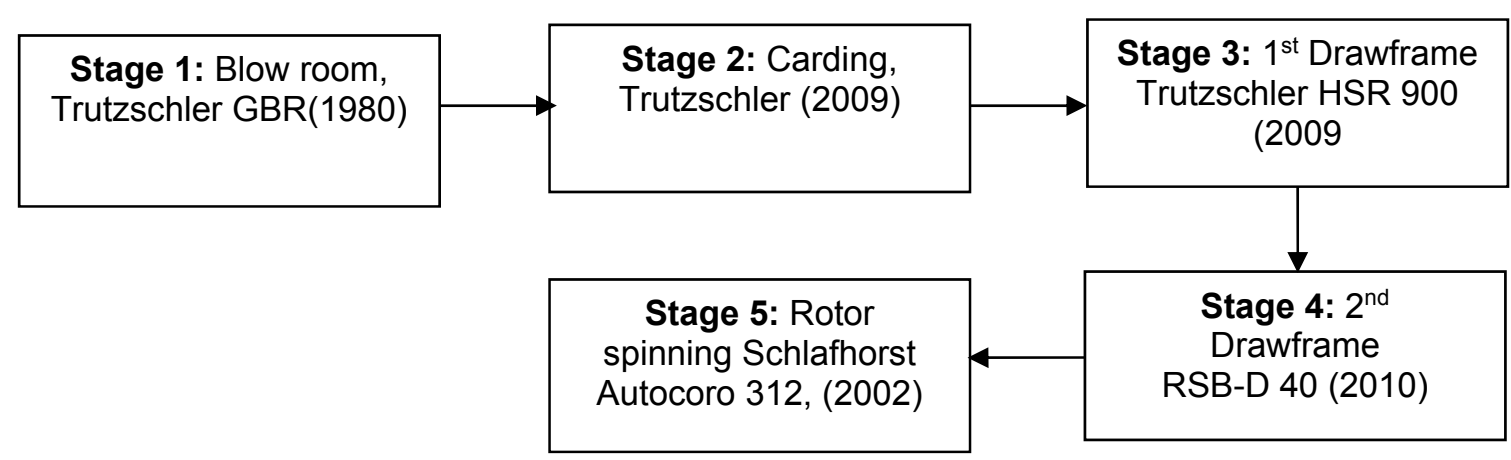

Figure 1. Cotton processing from blow room to rotor spinning

The effect of yarn count, yarn twist, opening roller speed and rotor speed on yarn tensile properties (strength and elongation) was investigated using statistical techniques. First the effect of single factor (while keeping the other three constant) on tensile properties was investigated. This was referred to as the effect of single factors on tensile properties.

Table 2. Machine and yarn parameters

\begin{tabular}{|c|c|c|c|l|}
\hline Experiment & Count & Twist & $\begin{array}{c}\text { Opening } \\
\text { Roller }\end{array}$ & $\begin{array}{c}\text { Rotor } \\
\text { speed }\end{array}$ \\
\hline 1 & 15 & 4.4 & 9000 & 80000 \\
\hline 2 & 15 & 4.4 & 8000 & 90000 \\
\hline 3 & 15 & 4.4 & 7000 & 100000 \\
\hline 4 & 15 & 5 & 9000 & 80000 \\
\hline 5 & 15 & 5 & 8000 & 90000 \\
\hline 6 & 15 & 5 & 7000 & 100000 \\
\hline 7 & 15 & 5.5 & 9000 & 90000 \\
\hline 8 & 15 & 5.5 & 8000 & 100000 \\
\hline 9 & 15 & 5.5 & 7000 & 80000 \\
\hline 10 & 24 & 4.4 & 9000 & 100000 \\
\hline 11 & 24 & 4.4 & 8000 & 80000 \\
\hline 12 & 24 & 4.4 & 7000 & 90000 \\
\hline 13 & 24 & 5 & 9000 & 90000 \\
\hline 14 & 24 & 5 & 8000 & 100000 \\
\hline 15 & 24 & 5 & 7000 & 80000 \\
\hline 16 & 24 & 5.5 & 9000 & 100000 \\
\hline 17 & 24 & 5.5 & 8000 & 80000 \\
\hline 18 & 24 & 5.5 & 7000 & 90000 \\
\hline
\end{tabular}


The effect of two factors (while keeping the other two factors constant) on yarn tensile properties was also investigated. This was referred to as the combined effect of factors on tensile properties. The effect of single and combined factors on yarn tensile properties was investigated using Analysis of variance (ANOVA) employing main effect plots and interaction plots.

\section{Results and discussions}

\subsection{Yarn strength}

\subsubsection{Effect of single variables on yarn strength}

The effect of single factors on yarn strength is given in Figure 2.This was studied by keeping the other three factors constant while studying one factor at a time.

It is clearly shown in Figure 2 that as the count increased from $15 \mathrm{Ne}$ to $24 \mathrm{Ne}$, yarn strength decreased. This may have been attributed to reduced yarn diameter with finer count $24 \mathrm{Ne}$. Ishtiaque et al [10] reported that an increase in count reduces the number of fibers in the yarn cross section. This may lead to poor spinning stability resulting into improper fiber alignment and yarn breakages which ultimately affects yarn strength.

With respect to twist, yarn strength is seen to increase with increase in twist from 4.4TM to 5TM beyond which it decreased. At low twist levels, there are no enough turns to compact the yarn very tightly together, hence lower twists are likely to produce yarn with lower strength.

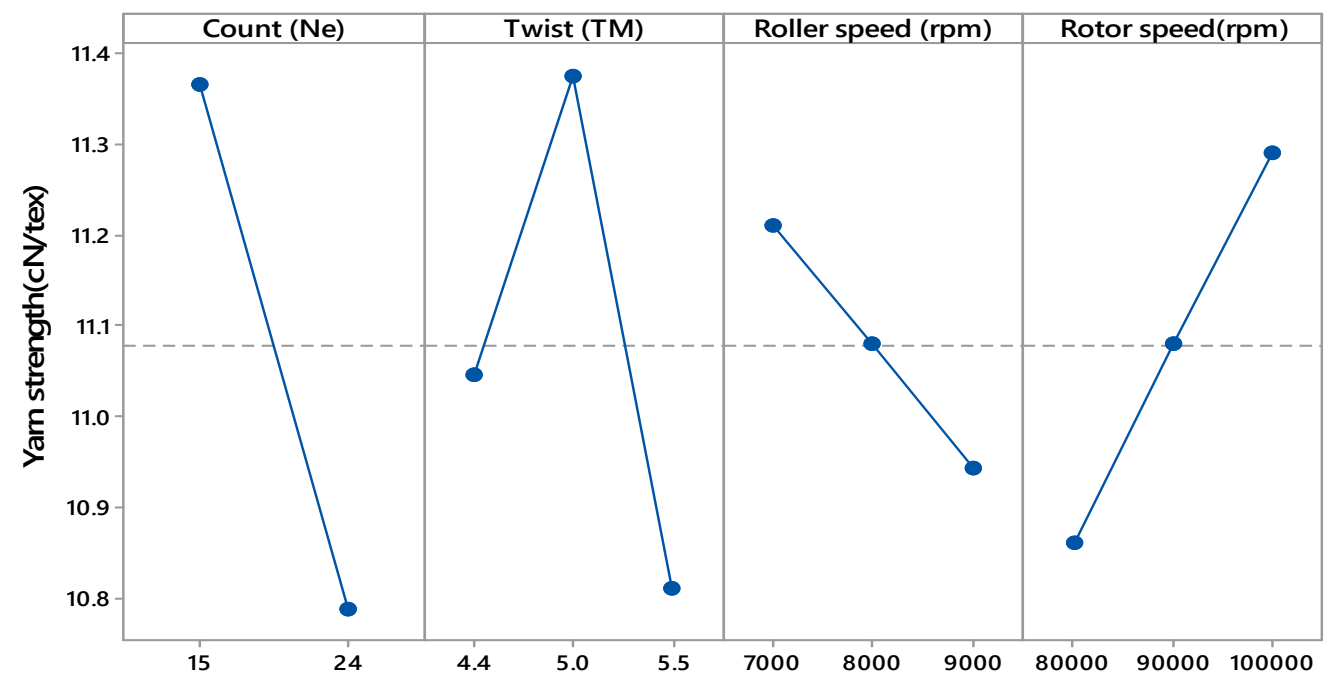

Figure 2. Effect of factor variables on single yarn strength

At slightly high twist levels, the inter fiber cohesion force increases preventing fiber slippage and hence contributing to yarn strength. However, very high twist levels on the other hand lead to poor twist propagation where the fibers making up the yarn loose contact with the twist angle during yarn formation causing breakages which may reduce yarn strength. For opening roller speed, an increase in speed from 7000-9000rpm led to a gradual decrease in yarn strength. Low opening roller speed are undesirable since inadequate fiber opening leads to poor yarn strength. While higher roller speeds contribute to better opening and hence proper straightening and individualization of fibers which leads to higher yarn strength, excessive opening roller speeds 
may lead to poor fiber orientation ultimately adversely affecting yarn tensile properties. With respect to rotor speed, increase in rotor speed increased yarn strength. Arain et al [3] stated that centrifugal forces increase with increase in rotor speed which in turn increases in the number of wrapper fibers. These wrapper fibers act as constriction and hence providing extra binding to the yarn which ultimately improves yarn strength. Excessive speeds however deteriorate fiber orientation which disturbs their strength transfer to the yarn hence causing a reduction in the yarn strength.

\subsubsection{Combined effect of factor variables on yarn strength}

The combined effect of spinning parameters on yarn strength was studied using interaction plots given in Figure 3. These were used to show the effect of two factors on yarn strength at different spinning level values while keeping the other two factors constant.

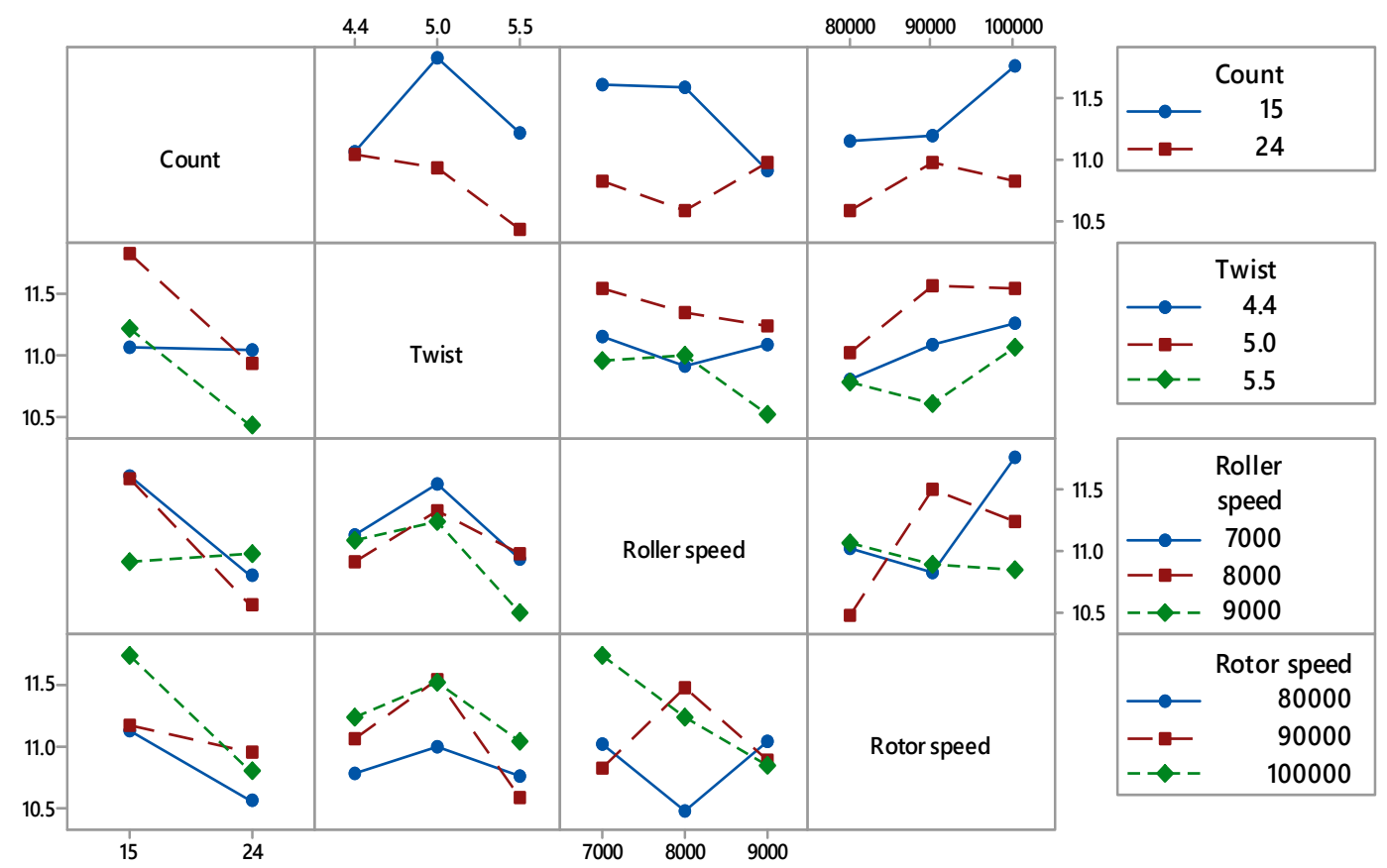

Figure 3. Combined effect of factor variables on yarn strength

When the effect of count was studied at varying levels of twist but at constant roller speed and rotor speed, an overlapping effect was observed when both counts ( $\mathrm{Ne} 15$ and $\mathrm{Ne} 24)$ gave a similar strength value at a twist multiplier value of 4.4TM. When the twist level increased to 5TM, yarn strength increased but later on decreased when the twist level was further increased to $5.5 \mathrm{TM}$ for $15 \mathrm{Ne}$ while $24 \mathrm{Ne}$ exhibited a decreasing trend. At very high twist levels, the increasing tension in the yarns would cause fiber breakages during yarn formation which reduces yarn strength.

At constant twist and rotor speed, the effect of count and opening roller speed showed that count $15 \mathrm{Ne}$ gave a constant strength value within the 7000- $8000 \mathrm{rpm}$ opening roller speed range beyond which it decreased while for count $24 \mathrm{Ne}$ the strength reduced in the 7000-8000rpm range but increased in the $8000-9000 \mathrm{rpm}$ range. At $9000 \mathrm{rpm}$ opening roller speeds, both counts gave a similar strength value, which was a reduced value for count $15 \mathrm{Ne}$, but an improved value for count $24 \mathrm{Ne}$. Khalilur and Habibur [7] results for rotor spun yarn of $16 \mathrm{Ne}$ were similar to our results for $15 \mathrm{Ne}$. 
The combined effect of count and rotor speed indicated that count $15 \mathrm{Ne}$ showed a slight increase in strength within $80,000-90,000 \mathrm{rpm}$ range and a very sharp increase in strength in the 90,000- 100,000 rpm range. With count $24 \mathrm{Ne}$, a sharp increase in yarn strength was observed in the $80,000-90,000 \mathrm{rpm}$ range and a reduction in the $90,000-100000 \mathrm{rpm}$ range. This may have been attributed to a larger yarn diameter and more fibers in the yarn cross section for coarse count $15 \mathrm{Ne}$ compared to the finer count $24 \mathrm{Ne}$ hence can withstand the high spinning tensions induced at higher rotor speeds. These results are quite similar to those obtained by Arain et al [3]where 90,000 rpm gave the best results in terms of strength.

When twist was studied in relation to other factors, the following was observed. At 4.4TM, strength is seen to be constant for both counts but at 5TM and 5.5TM, the strength was high for count $15 \mathrm{Ne}$ and low for count $24 \mathrm{Ne}$. At a constant count and rotor speed, twist levels were varied with opening roller speeds and it was observed that at $4.4 \mathrm{TM}$, yarn strength decreased in the 7000-8000 rpm opening roller speed range and then increased at 9000rpm. At 5TM, strength is seen to decrease with increasing roller speed while at $5.5 \mathrm{TM}$, an increase in opening roller speed led to an increase in strength up to 8000rpm beyond which it reduced as the speed of opening roller increased to $9000 \mathrm{rpm}$. Increasing twist leads to lower yarn production which may have contributed to better fiber opening by the opening roller due to prolonged time within the opening zone. This contributes to better fiber separation and orientation and could ultimately contribute to increased yarn strength. However, very high twist levels coupled with higher opening roller speed could lead to fiber rupture and hence a deterioration in yarn strength. Combination of twist and rotor speed showed that at 4.4TM, strength increased from 80,000 $\mathrm{rpm}$ to $100,000 \mathrm{rpm}$. At 5TM, strength increased steadily from 80,000 - 90,000 rpm after which it became constant within the $90000-100000 \mathrm{rpm}$ range. For a higher twist of 5.5TM, strength decreased in the $80,000-90,000 \mathrm{rpm}$ range beyond which it started to increase. This is unusual because for a given twist at higher rotor speeds, yarn delivery speed also increases which ultimately increases the feed rate at the opening roller region which deteriorates fiber individualization and orientation and hence leads to reduction of yarn strength.

When opening roller speed was considered with one more factor, while keeping the other two factors constant, the following was observed. For count $15 \mathrm{Ne}$, both 7000 and $8000 \mathrm{rpm}$ roller speeds gave a similar strength value. With count $24 \mathrm{Ne}$ in the same range, the strength reduced and the effect is more evident at 8000rpm. However, at 9000rpm yarn strength is almost constant for both counts. Varying twist and opening roller speed indicated that yarn strength increased with increase in roller speed within the 4.4-5TM range beyond which it reduced sharply in the 5-5.5TM range. This effect is more evident at $9000 \mathrm{rpm}$ which may be attributed to increased fiber rupture at the opening point. At 7000 and $9000 \mathrm{rpm}$, a similar strength value was noticed at 4.4TM and another at 7000 and $8000 \mathrm{rpm}$ at 5.5TM. For opening roller speed and rotor speed, it was found out that at $7000 \mathrm{rpm}$, yarn strength decreased with increase in rotor speed in the 80,000 - $90000 \mathrm{rpm}$ range probably due to insufficient fiber opening but later increased in the 90,000-100,000 rpm range. At $8000 \mathrm{rpm}$, strength increased sharply from $80000-90000 \mathrm{rpm}$ beyond which it started to decrease while at $9000 \mathrm{rpm}$, strength was seen to decrease from 80,000 - 90,000 rpm and then leveled off in the 90,000 $-100,000 \mathrm{rpm}$ range.

At a constant twist and opening roller speed, the effect of rotor speed and count was studied. At all rotor speed settings considered, yarn strength was observed to decrease with increase in count probably due to reduced number of fibers in the yarn cross section for the case of the finer count. At high rotor speeds, centrifugal forces increase hence increasing the spinning tension resulting into yarn breakages and ultimately a reduction in yarn strength with respect to count $24 \mathrm{Ne}$ [8]. When twist was considered, yarn strength increased with increase in both twist 
and rotor speed but beyond 5TM, it reduced. An overlapping interaction was also observed for 90,000 and $100,000 \mathrm{rpm}$ at 5TM. Combination of opening roller speed and rotor speed at constant count and twist showed that at 80,000 rpm, yarn strength decreased within the 7000 $8000 \mathrm{rpm}$ range but increased from 8000-9000rpm. Converse results were observed for 90,000 $\mathrm{rpm}$ rotor speed. At 100,000 rpm, yarn strength decreased with increase in opening roller speed from 7000-9000 rpm. Higher rotor speeds results into improper fiber straightening in the transportation tube and rotor groove due to short contact time before yarn formation resulting into reduced yarn strength. Ishtiaque et al [10] reported that increase in rotor speeds imposes powerful centrifugal forces on the fibers in the rotor groove. This leads to poor spinning stability hence an increase in yarn tension which in turn affects yarn strength. Soliman et al [11] stated that higher opening roller speeds could lead to good opening efficiency but at the same time weakens the fibers hence causing yarn breakages and deterioration in yarn strength and elongation. Therefore for optimum tensile properties, rotor speed and opening roller speeds need to be optimized.

From the optimization experiments, which considered yarn count, yarn twist, opening roller speed and rotor speed, it can concluded that count $15 \mathrm{Ne}$ coupled with a twist multiplier of $5 \mathrm{TM}$, an opening roller speed of $8000 \mathrm{rpm}$ and rotor speed of 90,000 rpm gave optimum strength results.

\subsection{Yarn elongation}

\subsubsection{Effect of single factor on yarn elongation}

The effect of single factor on elongation, while keeping the other factors constant was studied and the results are given in Figure 4, which indicated that yarn elongation decreased as the count increased from $15 \mathrm{Ne}-24 \mathrm{Ne}$ probably due to increased breakages resulting from spinning finer counts [10]. With respect to twist, elongation is seen to increase with an increase in twist up to $5 \mathrm{TM}$ beyond which there is a slight increase.

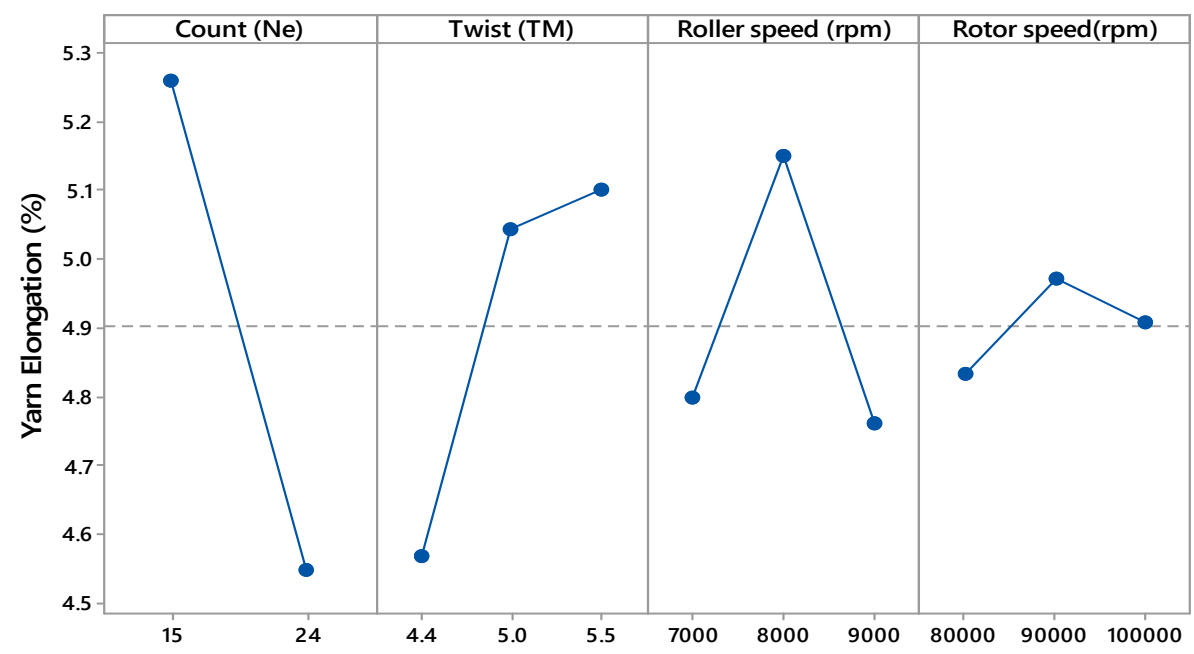

Figure 4. Influence of single variables on yarn elongation

At a high twist, the angle between the fiber spiral position and yarn axis increases which improves the springy behavior of fibers hence leading to higher elongation (Ahmed et al 2014, 
Arain et al 2012). For opening roller speed, elongation increased up to 8000rpm beyond which it decreased in the 8000-9000rpm range. This is due to better fiber opening which facilitates proper fiber individualization and straightening but excessive speeds instead lead to fiber breakages and hence a reduction in yarn elongation [7, 10]. Results for rotor speed showed that yarn elongation increased up to $900,000 \mathrm{rpm}$ and then decreased. At higher rotor speeds, centrifugal forces and spinning tension on the yarn increases which imparts a permanent strain on the yarn making it more compact and in so doing reduces its elongation [5].

\subsubsection{Combined effect of factors on yarn elongation}

The combined influence of two factors (while keeping the other two factors constant) on yarn elongation was studied using Figure 5.

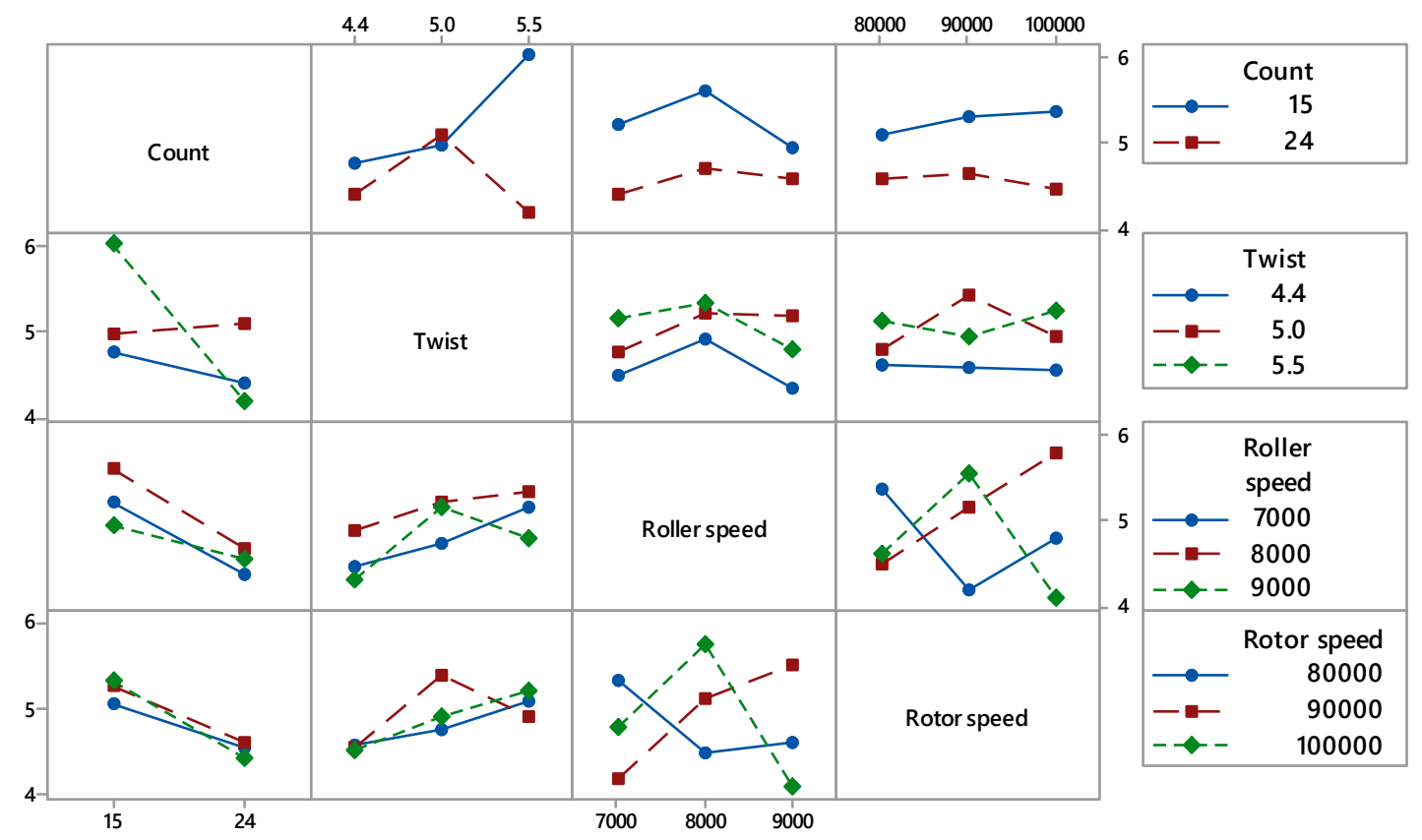

Figure 5. Combined effect of factor variables on yarn elongation

From the plots in Figure 5, it's evident that when count and twist were considered, yarn elongation increased with increase in twist for count $15 \mathrm{Ne}$ and an increase was observed for count $24 \mathrm{Ne}$ in the 4.4 -5TM range and then a decrease in the 5- 5.5TM range. In rotor spinning high twist values are recommended compared to ring spinning in order to get a better yarn but excessive twist levels distort the spinning stability causing breakages that reduce yarn elongation a case being more significant with finer counts. For count and opening roller speed, yarn elongation increased with increase in opening roller speed for both counts in the 7000$8000 \mathrm{rpm}$ range beyond which it decreased. With rotor speed, yarn elongation increased with increase in speed for both counts in the 80,000-90,000 rpm range beyond which it slightly leveled off for count $15 \mathrm{Ne}$ and decreased for count $24 \mathrm{Ne}$ probably due to increased yarn breakages resulting from increased spinning tensions. For twist at a constant opening roller and rotor speeds, it was found out that at 4.4TM, yarn elongation decreased with increase in count, at $5 \mathrm{TM}$ it increased slightly with increase in count while at 5.5TM, it decreased drastically with increase in count from 15-24 Ne possibly due to increased fiber breakages. Combined effect of twist and roller speed indicated that yarn elongation increased with increase in both factors but beyond $8000 \mathrm{rpm}$ it decreased at 4.4 and 5.5TM but leveled off at 5TM. Studying twist with 
rotor speed showed that at 4.4TM, an increase in rotor speed had no effect on yarn elongation. At 5TM, elongation increased with increase in rotor speed up to 90,000 rpm and then reduced while for 5.5TM, opposite results to those at 5TM were observed.

By varying opening roller speed and count at a constant twist and rotor speed, results showed that, any increase in opening roller speed led to a decrease in yarn elongation for a given increase in count from $15 \mathrm{Ne}-24 \mathrm{Ne}$. When twist and roller speed were varied, at 7000 and 8000 rpm any increase in twist led to an increase in cotton yarn elongation but at 9000rpm, elongation increased with twist from 4.4TM-5TM and then reduced. At high twist levels, fibers are assumed to spend more time in the opening roller region due to low production which could have led to improved fiber opening hence causing an increase in elongation. Higher roller speed and twist levels are however detrimental as they cause fiber rupture at the opening point which ultimately affects yarn elongation. The combined effect of opening roller speed and rotor speed, indicated that at $7000 \mathrm{rpm}$, elongation decreased with an increase in rotor speed up to 90,000 rpm and then increased. At $8000 \mathrm{rpm}$, an increas e in rotor speed led to an increase in elongation while at $9000 \mathrm{rpm}$, opposite results to those at 7000rpm were obtained.

Studying the combined effect of rotor speed and count showed that yarn elongation was high for count $15 \mathrm{Ne}$ at any value of rotor speed with an overlapping interaction at 90,000and $100,000 \mathrm{rpm}$ but it reduced with an increase of speed from count $15 \mathrm{Ne}-24 \mathrm{Ne}$ with an overlap at 80,000 and $90,000 \mathrm{rpm}$. This is because finer counts are susceptible to end breakages at high rotor speeds which ultimately reduced yarn elongation. Also, higher speeds create a permanent strain in the yarn due to higher spinning tension which in turn reduces yarn elongation. At varying twist levels and rotor speed, it was observed that there was a steady increase in elongation with an increase in twist and rotor speed at 80,000 rpm and 100,000 rpm. At 90,0 $00 \mathrm{rpm}$, elongation increased up to $5 \mathrm{TM}$ and then decreased. The combined effect of opening roller speed and rotor speed showed that at 80,000 rpm, elongation decreased from 7000-8000 rpm and then increased in the 8000-9000 rpm range, albeit at a lower rate. At 90,000 rpm, elongation increased steadily with increase in roller speed from 7000-9000 rpm while at $100,000 \mathrm{pm}$, it increased up to $8000 \mathrm{rpm}$ and then reduced with increase in roller speeds from $8000-9000 \mathrm{rpm}$.

After considering all the factors (count, twist, opening roller speed and rotor speed) the optimum parameters for yarn elongation were count of $15 \mathrm{Ne}$ with a twist multiplier of 5TM, opening roller speed of $8000 \mathrm{rpm}$ and rotor speed of $90,000 \mathrm{rpm}$. It is worthy noting that similar results were obtained for yarn tensile as reported early on.

\section{Conclusions}

In this study, the effect of yarn count, yarn twist, opening roller speed and rotor speed on yarn tensile properties (strength and elongation) was investigated. The effect of single factors showed that yarn strength decreased with increase in count and opening roller speed but increased with increase in twist and rotor speed while elongation decreased with increase in count but increased with increase in twist, roller speed and rotor speed up to certain specified values and then reduced. With respect to factor combinations, optimum tensile properties can be obtained by spinning count $15 \mathrm{Ne}$, at a twist multiplier of 5TM, opening roller speed of 8000 rpm and rotor speed of $90,000 \mathrm{rpm}$.

\section{References}


[1] Salhotra, KR. "An Overview of Spinning Technologies: Possibilities, Applications and Limitations." Indian Journal of Fibre and Textile Research, 17, no. 4(1992): 255-262.

[2] Lord, PR. Handbook of yarn production Technology, Science and Economics. New York Washington, DC: Woodhead Publishing Ltd, 2003.

[3] Arain, Farooq Ahmed, Tanwari, Anwaruddin, Hussain, Tanveer, Malik, Zulfiqar Ali. "Multiple response optimization of rotor yarn for strength, unevenness, hairiness and imperfections". Fibers and Polymers, 13, no.1 (2012): 118-122.

[4] Lawrence A. Carl. Fundamentals of Spun Yarn Technology. Boca Raton London New York Washington, D.C. CRC Press LLC, 2003.

[5] Gnanasekar K, Chellamani P., Karthikeyan S. "Influence of rotor speed in open-end spinning on yarn quality", Indian Journal of fibre \& Textile Research, 15, no. 4 (1990):164-168.

[6] Furter Richard. "Physical Properties of Spun Yarns". Uster Technology Application report, 2, no. 36 (2009): 23-24

[7] Khalilur Rahman Khan, Habibur Rahman, Khalilur RK, Habibur R. "Study of Effect of Rotor Speed, Combing-Roll Speed and Type of Recycled Waste on Rotor Yarn Quality Using Response Surface Methodology". Journal of Polymer and Textile Engineering, 2, no. (2015): 47-55.

[8] Ahmed F, Saleemi S, Rajput AW, Shaikh IA, Sahito AR. "Characterization of Rotor Spun Knitting Yarn at High Rotor Speeds". Technical Journal, University of Engineering and Technology (UET) Taxila, Pakistan, 19, no. 4 (2014) :73-78.

[9] Manohar JS, Rakshit AK, Balasubramanian N. "Influence of Rotor Speed, Rotor Diameter, and Carding Conditions on Yarn Quality in Open-End Spinning”. Textile Research Journal, 53, no. 8 (1983):497-503.

[10] Apurba Das, Saiyed Muzaffar Ishtiaque. "End Breakage In Rotor Spinning: Effect of Different Variables on Cotton Yarn End Breakage". Autex Research Journal, 4, no. 2 (2004): 52-59.

[11] Soliman HA, Hellwig AH. "Influence of Combing on Open-End Rotor Spinning Parameters.” International Textile Bulletin, Yarn and Fabric Forming, 2, (1996): 39-44. 